\title{
Nonsingular terminal sliding mode control of underwater remotely operated vehicles
}

\begin{tabular}{|c|c|}
\hline Journal: & Transactions of the Canadian Society for Mechanical Engineering \\
\hline Manuscript ID & TCSME-2017-0051.R1 \\
\hline Manuscript Type: & Article \\
\hline Date Submitted by the Author: & 20-Sep-2017 \\
\hline Complete List of Authors: & $\begin{array}{l}\text { Wang, Yaoyao; Nanjing University of Aeronautics \& Astronautics, College of } \\
\text { Mechanical and Electrical Engineering; Zhejiang University, the State Key } \\
\text { Laboratory of Fluid Power and Mechatronic Systems } \\
\text { Yan, Fei; Nanjing University of Aeronautics \& Astronautics, College of } \\
\text { Mechanical and Electrical Engineering } \\
\text { Tian, Bo; Nanjing University of Aeronautics \& Astronautics, College of } \\
\text { Mechanical and Electrical Engineering } \\
\text { Gu, Linyi; Zhejiang University, the State Key Laboratory of Fluid Power and } \\
\text { Mechatronic Systems } \\
\text { Chen, Bai; Nanjing University of Aeronautics and Astronautics, College of } \\
\text { Mechanical and Electrical Engineering }\end{array}$ \\
\hline $\begin{array}{r}\text { Is the invited manuscript for } \\
\text { consideration in a Special } \\
\text { Issue? : }\end{array}$ & N/A \\
\hline Keywords: & $\begin{array}{l}\text { nonsingular terminal sliding mode control, adaptive nonsingular terminal } \\
\text { sliding mode control, remotely operated vehicles, Lyapunov stability }\end{array}$ \\
\hline
\end{tabular}




\title{
NONSINGULAR TERMINAL SLIDING MODE CONTROL OF UNDERWATER REMOTELY OPERATED VEHICLES
}

\author{
Yaoyao Wang ${ }^{1,2 \dagger}$, Fei Yan $^{1}$, Bo Tian ${ }^{1}$, Linyi Gu², Bai Chen ${ }^{1}$ \\ ${ }^{1}$ College of Mechanical \& Electrical Engineering, Nanjing University of Aeronautics \& Astronautics, \\ Nanjing, China. \\ ${ }^{2}$ the State Key Laboratory of Fluid Power and Mechatronic Systems, Zhejiang University, Hangzhou, \\ China \\ E-mail: yywang_cmee@nuaa.edu.cn (Corresponding author:Dr.Wang) \\ Received Month 0000, Accepted Month 0000 \\ No. 00-CSME-00, E.I.C. Accession Number 0000
}

\begin{abstract}
This study investigates the nonsingular terminal sliding mode control (NTSMC) method for the 4DOF (degrees of freedom) trajectory tracking control problem of underwater remotely operated vehicles (ROVs) in the presence of parametric uncertainties and external disturbances. Two new control algorithms have been developed for ROVs. The first one, combining a nonsingular sliding surface with a fast-TSM-type reaching law, is nonsingular and chattering-free. The second one, introducing adaptive methodology to compensate for the lumped uncertainties, is an improved version of the first algorithm and can be called adaptive NTSMC (ANTSMC). The application of adaptive methodology effectively eases the chattering problem. Meanwhile, it also provides better robustness and higher tracking precision compared with the first algorithm. A corresponding stability analysis is presented using Lyapunov stability theory, and some comparative numerical simulation results are presented to show the effectiveness of the proposed approaches.
\end{abstract}

Keywords: nonsingular terminal sliding mode control; adaptive nonsingular terminal sliding mode control; remotely operated vehicles; Lyapunov stability 


\section{INTRODUCTION}

In recent decades, underwater remotely operated vehicles (ROVs) have become significantly important in the area of underwater scientific and commercial investigations due to their great adaptability and working capabilities in the deep sea. As the field of ROV application expands, fast dynamic response and high-precision tracking control performance are required for more and more practical applications. However, designing a proper control system for a ROV is still a great engineering challenge due to the nonlinearity and unpredictable external disturbances, as well as the difficulties in accurately modeling the hydrodynamic effect (Wang et al., 2016a).

The sliding mode control (SMC) method, one of the most exciting achievements in the control community, is an effective tool to handle the aforementioned control problems. There are two main parts in the SMC method: one is the driving part, which forces the trajectory of the system to reach and stay on a stable sliding surface; the other is a sliding surface that guarantees the desired system dynamics and obtains robustness against lumped uncertainties because the system is insensitive to all types of disturbances when it remains on the sliding surface (Baek et al., 2016).

Therefore, the SMC methodology has been widely used in the trajectory tracking control of underwater vehicles, including the underwater ROVs (Yan et al., 2015; Cui et al., 2017; Chu et al 2017). By adopting the adaptive methodology to estimate the bounds of the lumped uncertainties, a robust adaptive sliding mode controller is proposed for ROVs (Hoang and Kreuzer 2008). Using an adaptive fuzzy algorithm for uncertainty/disturbance compensation, a novel adaptive fuzzy sliding mode controller is presented for the depth control of ROVs (Bessa et al., 2008). Combining the neural-network strategy and sliding mode control method, a sliding-mode neural-network scalar (SMNNS) control system is proposed for the 4-DOF (degrees of freedom) control of ROVs (Bagheri and Moghaddam 2009a, 2009b). Synthesizing the adaptive neuro-fuzzy strategy and genetic algorithm and sliding mode algorithm, an adaptive neuro-fuzzy sliding-mode-based genetic algorithm (ANFSGA) control system is presented for the 4-DOF control of ROVs (Javadi-Moghaddam and Bagheri 2010a, 2010b). Their 
effectiveness and high-precision are validated through numerical simulation experiments. By expanding the control method proposed in (Bessa et al., 2008) to multi-degree of freedom (MDOF) area, an adaptive fuzzy sliding mode controller for ROVs is presented (Bessa et al., 2010). Using the local recurrent neural network to estimate the unknown part of the affine system online, a local recurrent neural network based sliding mode controller is proposed for underwater robots (Zhang and Chu 2012a, 2012b). And its effectiveness is validated through tracking experiments of the experimental prototype of underwater robot. To summarize the aforementioned works, the studies of SMC method for ROVs are mainly focused on the traditional linear-hyperplane based SMC method, which means that the control system tracking error need infinite time to converge to zero.

However, it is widely known that finite-time stabilization of dynamical systems may lead to a highprecision performance besides finite-time convergence ( $\mathrm{Yu}$ et al., 2005). Therefore, a new control methodology and its improved version called terminal sliding mode control (TSMC) and nonsingular terminal sliding mode control (NTSMC) were proposed (Feng et al., 2002; Yu and Man 1998a, 1998b). They can achieve finite-time convergence by replacing the linear sliding surface with a nonlinear one which can be called terminal sliding mode (TSM). Attracted by its obvious advantages, lots of scholars and engineers have been devoted themselves to develop the TSMC/NTSMC method for all kinds practical applications. It has been applied in many practical systems, such as manipulators (Wang et al, 2016b, 2016c), underwater vehicle-manipulator system (Wang et al, 2017), multiple mobile robots (Zhao and Zou 2012a, 2012b), spacecraft(Wang and Sun 2012a, 2012b) and so on.

However, to the best of the authors' knowledge, there are few studies that combine the nonlinear sliding surface dynamic with the ROV' dynamics for the trajectory tracking control of a ROV. Therefore, inspired by the achievements of the aforementioned studies, we present two new control laws for the control problem of ROVs using TSMC methodology. Both of the proposed methods can obtain highprecision tracking performance and great robustness against lumped uncertainties. The effectiveness of these proposed methods have been validated through numerical simulation experiments. 
The rest of the paper is organized as follows. In section 2, ROVs' dynamic model is presented. In section 3, the main results are presented. And two control laws using NTSMC methodology are proposed and analyzed. In section 4, some simulations results are given to validate the effectiveness of the proposed methods. Finally, some conclusions are presented in section 5.

\section{ROV'S DYNAMIC MODEL}

A reasonable dynamic model to describe ROVs' behavior must contain both the rigid-body dynamics of the vehicle's body and the representation of the surrounding fluid dynamics (Bessa et al., 2010). Thus, the dynamic model of a ROV is often described with respect to earth-fixed frame and body-fixed frame, as shown in Fig.1.

Before presenting the dynamic model of a ROV, some related assumptions must be noted(Zhu and $\mathrm{Gu}$ 2011a, 2011b):

Assumption 1. The ROV's center of gravity coincides with the origin of the body-fixed frame, and it is also in the same vertical line with the buoyancy center, which means $x_{\mathrm{B}}=y_{\mathrm{B}}=0$.

Assumption 2. The system is stable with respect to roll and pitch angles, which means that the angles and angular velocity of roll and pitch can be regarded as zero.

Assumption 3. All of the off-diagonal elements of the inertial matrix are very small compared to the diagonal ones. Thus, the inertial matrix can be regarded as a diagonal matrix.

Remark 1. In practical applications, above three assumptions usually can be easily satisfied to some extent for the control purpose of ROVs.

Then, the simplified dynamic model of a ROV in 4-DOF can be represented as (Zhu et al., 2011; Fossen et al., 1994):

$$
\begin{aligned}
& M \dot{v}+C(v) v+D(v) v+g(\eta)+\tau_{d}=\tau . \\
& \dot{\eta}=J(\eta) v .
\end{aligned}
$$




$$
\tau=B u
$$

where $\quad M=M_{R B}+M_{A}, C(v)=C_{R B}(v)+C_{A}(v), D(v)=D_{L}+D_{Q}(v) . \quad M_{R B} \in R^{4 \times 4} \quad$ and $\quad C_{R B}(v) \in R^{4 \times 4}$ represent the rigid body inertial matrix and the Coriolis and centripetal matrix, respectively. $M_{A} \in R^{4 \times 4}$ and $C_{A}(v) \in R^{4 \times 4}$ denote the added mass matrix and the added Coriolis and centripetal matrix, respectively. $D_{L} \in R^{4 \times 4}$ and $D_{Q} \in R^{4 \times 4}$ denote the linear and quadratic drag matrices, respectively. The vector $g(\eta) \in R^{4 \times 1}$ is the combined force/moment of gravity and buoyancy in the body-fixed frame. Moreover, $\eta=[x, y, z, \psi]^{T}$ represents the ROV's position and orientation in the earth-fixed frame, and $v=[u, v, w, r]^{T}$ represents the ROV's linear and angular velocity in the body-fixed frame. $\tau_{d} \in R^{4 \times 1}$ denotes the disturbance force/moment vector, and $\tau \in R^{4 \times 1}$ denotes the system input produced by the propellers. $J(\eta) \in R^{4 \times 4}$ is the kinematic transformation matrix which expresses the relationship between the body-fixed frame and the earth-fixed frame, and can be written as follows

$$
J(\eta)=\left[\begin{array}{cccc}
\cos \psi & -\sin \psi & 0 & 0 \\
\sin \psi & \cos \psi & 0 & 0 \\
0 & 0 & 1 & 0 \\
0 & 0 & 0 & 1
\end{array}\right]
$$

$B \in R^{4 \times 5}$, the dynamic distribution matrix, is mainly determined by the arrangement of the ROV's propellers as shown in Fig.2. $u \in R^{5 \times 1}$ is the force vector produced by the propellers.

Furthermore, equation (1) can be expressed in another form for the convenience of controller design:

$$
M_{0} \dot{v}+C_{0}(v) v+D_{0}(v) v+g_{0}(\eta)=\tau+F
$$

where $M_{0}, C_{0}(v), D_{0}(v), g_{0}(\eta)$ are the nominal parameter matrices, and $F=-\left(\tau_{d}+\Delta M \dot{v}+\Delta C(v) v+\right.$ $\Delta D(v) v+\Delta g(\eta))$ is the lumped disturbance force/moment vector including parametric uncertainties and external disturbances. 
Finally, the other simplified parameter matrices can be written as:

$$
\begin{aligned}
& M_{0}=\operatorname{diag}\left\{m_{v}-X_{\dot{u}}, m_{v}-Y_{\dot{v}}, m_{v}-Z_{\dot{w}}, I_{z}-N_{\dot{r}}\right\} . \\
& C_{0}(v)=\left[\begin{array}{cccc}
0 & 0 & 0 & -\left(m_{v}-Y_{\dot{v}}\right) v \\
0 & 0 & 0 & -\left(m_{v}-X_{\dot{u}}\right) u \\
0 & 0 & 0 & 0 \\
\left(m_{v}-Y_{\dot{v}}\right) v & -\left(m_{v}-X_{\dot{u}}\right) u & 0 & 0
\end{array}\right] . \\
& D_{0}(v)=\operatorname{diag}\left\{X_{u}+X_{u|u|} u, Y_{v}+Y_{v|v|} v, Z_{w}+Z_{w|w|} w, N_{r}+N_{r|r| r} r\right\} \\
& B=\left[\begin{array}{ccccc}
0,0, W-B, 0]^{T} . & -\cos \theta & 0 \\
\cos \theta & \cos \theta & -\cos \theta & -\sin \theta & 0 \\
-\sin \theta & \sin \theta & \sin \theta & -\ln \\
0 & 0 & 0 & 0 & 1 \\
-L & L & -L & L & 0
\end{array}\right] .
\end{aligned}
$$

where $W$ and $B$ represent the ROV's weight and buoyancy respectively. And $\theta=30^{\circ}, L=0.1 \mathrm{~m}$.

\section{CONTROL SCHEME DESIGN}

In this subsection, two control methods using NTSMC methodology are presented and analyzed, and the corresponding stability analysis is also given in detail. Some preliminary information must be presented prior to the control algorithm design and analysis (Yu et al., 2005; Zhu et al., 2011).

Assumption 4. The lumped disturbance force/moment vector $F$ is bounded, which means that there exist some positive numbers $\rho_{0}, \rho_{1}, \rho_{2}, f$ such that

$$
\left\|J(\eta) M_{0}^{-1} F\right\|<\rho_{0}+\rho_{1}\|e\|+\rho_{2}\|\dot{e}\| \leq f<+\infty .
$$

Assumption 5. The desired trajectory $\eta_{d}$ is smooth, i.e., $\dot{\eta}_{d}, \ddot{\eta}_{d}$ exist and are bounded. 
Definition 1. Consider the nonlinear system $\dot{x}=f(x, u)$, where $x$ is the state vector and $u$ is the input vector. The corresponding solution is called practical finite-time stable (PFS) if for all $x\left(t_{0}\right)=x_{0}$, there exist $\delta>0$ and $T\left(\delta, x_{0}\right)<\infty$, such that $\|x(t)\|<\delta$, for all $t \geq t_{0}+T$.

Lemma 1. For any real positive numbers $x_{i}, i=1, \cdots n$ and $0 \leq a \leq 1,0<b<2$, then the following two inequalities hold:

$$
\begin{aligned}
& \left(x_{1}+\cdots+x_{n}\right)^{a} \leq x_{1}^{a}+\cdots+x_{n}{ }^{a} \\
& \left(x_{1}^{2}+\cdots+x_{n}{ }^{2}\right)^{b} \leq\left(x_{1}^{b}+\cdots+x_{n}^{b}\right)^{2}
\end{aligned}
$$

Lemma 2. An extended Lyapunov description of finite-time stability can be given with a form of fast TSM as

$$
V \dot{(x)}+\alpha V(x)+\beta V^{\gamma}(x) \leq 0, \alpha>0, \beta>0,0<\gamma<1
$$

and the settling time can be given by

$$
T \leq \frac{1}{\alpha(1-\gamma)} \ln \frac{\alpha V^{1-\gamma}\left(x_{0}\right)+\beta}{\beta} .
$$

Lemma 3. For any three real numbers $x_{1}, x_{2}, x_{3}$, if $x_{1}+x_{2}=x_{3}$, then for any positive scalar $a \geq 0.5$, the following inequality holds:

$$
x_{1} x_{2} \leq-\frac{2 a-1}{2 a} x_{1}^{2}+\frac{a}{2} x_{3}^{2}
$$

Lemma 4. Consider the system $\dot{x}=f(x, u)$. Suppose that there exists a continuous function $V(x)$, scales $\lambda>0,0<\mu<1$ and $0<\omega<\infty$ such that

$$
\dot{V}(x) \leq-\lambda V^{\mu}(x)+\omega
$$


Then, the system $\dot{x}=f(x, u)$ is PFS. Additionally, the state trajectory of the closed-loop system is bounded in finite time as:

$$
\lim _{a \rightarrow a_{0}} x \in\left(V^{\mu}(x) \leq \frac{\omega}{\left(1-a_{0}\right) \lambda}\right)
$$

where $0<a_{0}<1$. The time needed for the state $x$ to reach the area (18) is bounded as:

$$
T \leq \frac{V^{1-\mu}\left(x_{0}\right)}{\lambda a_{0}(1-\mu)}
$$

where $V\left(x_{0}\right)$ is the initial value of $V(x)$.

For simplicity, the following notations will be adopted: the operation $<,>, \leq, \geq$ for two vectors is performed in terms of corresponding elements, $x_{i}$ denotes the i-th element of vector $x$, and $\operatorname{sig}(\boldsymbol{x})^{\gamma}=\left[\left|x_{1}\right|^{\gamma_{1}} \operatorname{sign}\left(x_{1}\right), \ldots,\left|x_{n}\right|^{\gamma_{n}} \operatorname{sign}\left(x_{n}\right)\right]^{T}, \boldsymbol{x}^{\gamma}=\left[x_{1}^{\gamma_{1}}, \ldots, x_{n}^{\gamma_{n}}\right]^{T},|\boldsymbol{x}|^{\gamma}=\left[\left|x_{1}\right|^{\gamma_{1}}, \ldots,\left|x_{n}\right|^{\gamma_{n}}\right]^{T}$.

\subsection{Nonsingular Terminal Sliding Mode Control Method Design}

Inspired by (Yu et al., 2005), a NTSMC method for the 4-DOF trajectory tracking control of ROVs is proposed. This method is similar to the one presented in ( $\mathrm{Yu}$ et al., 2005), with the exception that different dynamic model and reaching law are adopted in this case.

Define the tracking error as

$$
e=\eta-\eta_{d}
$$

Then, a NTSM and a fast-TSM-type reaching law are defined as (Yu et al., 2005)

$$
\begin{aligned}
& \boldsymbol{s}=\boldsymbol{e}+\boldsymbol{\beta} \operatorname{sig}(\dot{\boldsymbol{e}})^{\sigma} . \\
& \dot{\boldsymbol{s}}=-\operatorname{diag}\left(|\dot{\boldsymbol{e}}|^{\sigma-1}\right)\left[\boldsymbol{k}_{1} \boldsymbol{s}+\boldsymbol{k}_{2} \operatorname{sig}(\boldsymbol{s})^{\kappa}\right] .
\end{aligned}
$$


where $\sigma=p / q, \kappa=m / n$ and $p_{i}, q_{i}, m_{i}, n_{i}$ are positive odd numbers, and satisfy the following inequality $1<\sigma_{i}<2,0<\kappa_{i}<1 . \boldsymbol{\beta}=\operatorname{diag}\left(\beta_{1}, \ldots, \beta_{4}\right), \beta_{i} \in \mathrm{R}^{+}, \boldsymbol{s}=\left[s_{1}, \ldots, s_{4}\right]^{T}, \boldsymbol{e}, \dot{\boldsymbol{e}} \in \mathrm{R}^{4 \times 1}$.

Remark 2. The reaching law adopted here is slightly different from the one from (Yu et al., 2005), which can be written as $\dot{\boldsymbol{s}}=-\boldsymbol{k}_{1} \boldsymbol{s}-\boldsymbol{k}_{2} \operatorname{sig}(\boldsymbol{s})^{\kappa}$. At first glance, it seems that the latter may provide better dynamic performance. However, in the following proof procedure of Theorem 1, it can clearly be observed that the term $|\dot{\boldsymbol{e}}|^{\sigma-1}$ is essential for achieving the nonsingularity of the closed-loop system.

Then, the following theorem can be obtained.

Theorem 1. For the 4-DOF simplified dynamic model (5), if assumptions $1 \sim 5$ hold, and the NTSM and reaching law are chosen as (21)(22), respectively. Then if the controller is designed as follows

$$
\begin{aligned}
\boldsymbol{\tau} & =\boldsymbol{\tau}_{1}+\boldsymbol{\tau}_{2}, \\
\boldsymbol{\tau}_{1} & =\boldsymbol{M}_{0} \boldsymbol{J}^{-1}(\boldsymbol{\eta})\left[\ddot{\boldsymbol{\eta}}_{\mathrm{d}}-\boldsymbol{J}(\boldsymbol{\eta}) \boldsymbol{v}\right]+\boldsymbol{C}_{0}(\boldsymbol{v}) \boldsymbol{v}+\boldsymbol{D}_{0}(\boldsymbol{v}) \boldsymbol{v}+\boldsymbol{g}_{0}(\boldsymbol{\eta}), \\
\boldsymbol{\tau}_{2} & =-\boldsymbol{M}_{0} \boldsymbol{J}^{-1}(\boldsymbol{\eta}) \boldsymbol{\tau}_{0}, \\
\boldsymbol{\tau}_{0} & =\operatorname{diag}\left(\boldsymbol{\beta}^{-1} \sigma^{-1}\right)\left[\boldsymbol{k}_{1} \boldsymbol{s}+\boldsymbol{k}_{2} \operatorname{sig}(\boldsymbol{s})^{\kappa}+\operatorname{sig}(\dot{\boldsymbol{e}})^{2-\sigma}\right] .
\end{aligned}
$$

The trajectory of the system will converge to the following ball field in finite time

$$
\begin{gathered}
\|\boldsymbol{s}\| \leq \Delta=\min \left\{\Delta_{1}, \Delta_{2}\right\}, \\
\Delta_{1}=\left\|\operatorname{diag}(\boldsymbol{\beta} \sigma) \boldsymbol{J}(\boldsymbol{\eta}) \boldsymbol{M}_{0}^{-1}\right\| \rho(\boldsymbol{e}, \dot{\boldsymbol{e}}) / \lambda_{\min }\left(\boldsymbol{k}_{1}\right), \Delta_{2}=\left(\left\|\operatorname{diag}(\boldsymbol{\beta} \sigma) \boldsymbol{J}(\boldsymbol{\eta}) \boldsymbol{M}_{0}^{-1}\right\| \rho(\boldsymbol{e}, \dot{\boldsymbol{e}}) / \lambda_{\min }\left(\boldsymbol{k}_{2}\right)\right)^{1 / \kappa} .
\end{gathered}
$$

Moreover, the position and velocity tracking errors will converge to the following field in finite time

$$
\left|e_{i}\right| \leq \Delta_{e_{i}}=2 \Delta,\left|\dot{e}_{i}\right| \leq \Delta_{\dot{e}_{i}}=\left(\beta_{i}^{-1} \Delta\right)^{1 / \sigma_{i}}
$$

where $\rho(\boldsymbol{e}, \dot{\boldsymbol{e}})=\rho_{0}+\rho_{1}\|\boldsymbol{e}\|+\rho_{2}\|\dot{\boldsymbol{e}}\|, i=1 \sim 4$.

Proof. The proof procedure is similar to the one that is presented in (Yu et al., 2005; Wang et al., 2013). Choose a Lyapunov function as 


$$
V=\frac{1}{2} s^{T} s
$$

By differentiating $V$ with respect to time and substituting control law(23), we have

$$
\dot{V}=\boldsymbol{s}^{T} \dot{\boldsymbol{s}}=-\boldsymbol{s}^{T} \operatorname{diag}\left(|\dot{\boldsymbol{e}}|^{\sigma-1}\right)\left\{\boldsymbol{k}_{1} \boldsymbol{s}+\boldsymbol{k}_{2} \operatorname{sig}(\boldsymbol{s})^{\kappa}-\operatorname{diag}(\boldsymbol{\beta} \sigma) \boldsymbol{J}(\boldsymbol{\eta}) \boldsymbol{M}_{0}^{-1} \boldsymbol{F}\right\}
$$

Eq.(27) can be rewritten in the following two forms

$$
\begin{aligned}
& \dot{V}=-\boldsymbol{s}^{T} \operatorname{diag}\left(|\dot{\boldsymbol{e}}|^{\sigma-1}\right)\left\{\left(\boldsymbol{k}_{1}-\operatorname{diag}(\boldsymbol{D}) \times \operatorname{diag}^{-1}(\boldsymbol{s})\right) \boldsymbol{s}+\boldsymbol{k}_{2} \operatorname{sig}(\boldsymbol{s})^{\kappa}\right\} \\
& \dot{V}=-\boldsymbol{s}^{T} \operatorname{diag}\left(|\dot{\boldsymbol{e}}|^{\sigma-1}\right)\left\{\boldsymbol{k}_{1} \boldsymbol{s}+\left(\boldsymbol{k}_{2}-\operatorname{diag}(\boldsymbol{D}) \times \operatorname{diag}^{-1}\left(\operatorname{sig}(\boldsymbol{s})^{\kappa}\right)\right) \operatorname{sig}(\boldsymbol{s})^{\kappa}\right\} .
\end{aligned}
$$

where $\boldsymbol{D}=\operatorname{diag}(\boldsymbol{\beta} \sigma) \boldsymbol{J}(\boldsymbol{\eta}) \boldsymbol{M}_{0}^{-1} \boldsymbol{F} \in \mathrm{R}^{4 \times 1}$.

Eq.(28) can be further rewritten as

$$
\dot{V}=-\boldsymbol{s}^{T} \overrightarrow{\boldsymbol{k}}_{1} \boldsymbol{s}-\boldsymbol{s}^{T} \overrightarrow{\boldsymbol{k}}_{2} \operatorname{sig}(\boldsymbol{s})^{K}
$$

where $\overrightarrow{\boldsymbol{k}}_{1}=\operatorname{diag}\left(|\dot{\boldsymbol{e}}|^{\sigma-1}\right)\left(\boldsymbol{k}_{1}-\operatorname{diag}(\boldsymbol{D}) \times \operatorname{diag}^{-1}(\boldsymbol{s})\right), \overrightarrow{\boldsymbol{k}}_{2}=\operatorname{diag}\left(|\dot{\boldsymbol{e}}|^{\sigma-1}\right) \boldsymbol{k}_{2}$.

When $\dot{e} \neq 0$ and $\lambda_{\text {min }}\left(\overrightarrow{\boldsymbol{k}}_{1}\right)>0, \lambda_{\min }\left(\overrightarrow{\boldsymbol{k}}_{2}\right)>0$, according to inequality (13) from Lemma 1 , we have

$$
\dot{V}=-\boldsymbol{s}^{T} \overrightarrow{\boldsymbol{k}}_{1} \boldsymbol{s}-\boldsymbol{s}^{T} \overrightarrow{\boldsymbol{k}}_{2} \operatorname{sig}(\boldsymbol{s})^{\kappa} \leq-2 \lambda_{\min }\left(\overrightarrow{\boldsymbol{k}}_{1}\right) V-2^{(\kappa+1) / 2} \lambda_{\min }\left(\overrightarrow{\boldsymbol{k}}_{2}\right) V^{(\kappa+1) / 2}
$$

where $\lambda_{\min }\left(\overrightarrow{\boldsymbol{k}}_{1}\right), \lambda_{\min }\left(\overrightarrow{\boldsymbol{k}}_{2}\right)$ are the smallest characteristic root of $\overrightarrow{\boldsymbol{k}}_{1}, \overrightarrow{\boldsymbol{k}}_{2}$.

Then, according to Lemma 2, the settling time can be calculated as

$$
T_{r} \leq \frac{1}{\lambda_{\text {min }}\left(\overrightarrow{\boldsymbol{k}}_{1}\right)(1-\kappa)} \ln \left(\frac{2 \lambda_{\text {min }}\left(\overrightarrow{\boldsymbol{k}}_{1}\right) V^{\frac{1-\kappa}{2}}\left(x_{0}\right)}{2^{\frac{\kappa+1}{2}} \lambda_{\text {min }}\left(\overrightarrow{\boldsymbol{k}}_{2}\right)}+1\right) .
$$

The trajectory of the system will converge to the following ball field in finite time

$$
\|s\| \leq\left\|\operatorname{diag}(\boldsymbol{\beta} \sigma) \boldsymbol{J}(\boldsymbol{\eta}) \boldsymbol{M}_{0}^{-1}\right\| \rho(\boldsymbol{e}, \dot{\boldsymbol{e}}) / \lambda_{\min }\left(\boldsymbol{k}_{1}\right)
$$


A similar procedure can be conducted for(29), and we have that the trajectory of the system will converge to the following ball filed in finite time

$$
\|S\| \leq\left(\left\|\operatorname{diag}(\boldsymbol{\beta} \sigma) \boldsymbol{J}(\boldsymbol{\eta}) \boldsymbol{M}_{0}^{-1}\right\| \rho(\boldsymbol{e}, \dot{\boldsymbol{e}}) / \lambda_{\min }\left(\boldsymbol{k}_{2}\right)\right)^{1 / \kappa}
$$

Combining (33) and(34), we have that the trajectory of the system will converge to the field (24) in finite time.

Note that $\dot{e}_{i}=0$ may hinder the reachability of $\operatorname{NFTSM}(21)$, however, we will prove that this situation will not occur Substituting the control law (23) into (5) and combining this with the fact that $\dot{e}_{i}=0$, we have

$$
\ddot{e}_{i}=-\beta_{i}^{-1} \sigma_{i}^{-1}\left[k_{1 i} s_{i}+k_{2 i} \operatorname{sig}\left(s_{i}\right)^{\kappa_{i}}-D_{i}\right] .
$$

When $s_{i} \notin \Delta$, we have

$$
\begin{aligned}
& \ddot{e}_{i}=-\beta_{i}^{-1} \sigma_{i}^{-1}\left[\left(k_{1 i}-D_{i} s_{i}^{-1}\right) s_{i}+k_{2 i} \operatorname{sig}\left(s_{i}\right)^{\kappa_{i}}\right] \neq 0 . \\
& \ddot{e}_{i}=-\beta_{i}^{-1} \sigma_{i}^{-1}\left[k_{1 i} s_{i}+\left(k_{2 i}-D_{i} \operatorname{sig}\left(s_{i}\right)^{-\kappa_{i}}\right) \operatorname{sig}\left(s_{i}\right)^{\kappa_{i}}\right] \neq 0 .
\end{aligned}
$$

Thus, the finite-time reachability of NTSM (21) can be still assured. Combining (21) and (24) yields

$$
s_{i}=e_{i}+\beta_{i} \operatorname{sig}\left(\dot{e}_{i}\right)^{\sigma_{i}}<\Delta_{i}
$$

Inequality (37) can be rearranged as

$$
e_{i}+\left(\beta_{i}-s_{i} \operatorname{sig}\left(\dot{e}_{i}\right)^{-\sigma_{i}}\right) \operatorname{sig}\left(\dot{e}_{i}\right)^{\sigma_{i}}=0
$$

It is obvious that, if $\beta_{i}-s_{i} \operatorname{sig}\left(\dot{e}_{i}\right)^{-\sigma_{i}}>0$, inequality (38) still kept in the form of TSM. Thus, it can be yielded that the velocity tracking error of the closed-loop system will converge to the following ball field in finite time

$$
\left|\dot{e}_{i}\right| \leq \Delta_{\dot{e}_{i}}=\left(\beta_{i}^{-1} \Delta\right)^{1 / \sigma_{i}}
$$


Combining inequality (39) with the definition of TSM (21) yields

$$
\left|e_{i}\right| \leq\left|s_{i}\right|+\beta_{i}\left|\dot{e}_{i}\right|^{\sigma_{i}} \leq 2 \Delta=\Delta_{e_{i}}
$$

Therefore, the position and velocity tracking errors will converge to the ball field (25) in finite time. This completes the proof.

Remark 3. As indicated in the proposed controller(23), the power exponents $\kappa$ and $2-\sigma$ are both positive, therefore the singularity problem has been effectively settled.

Remark 4. As shown in inequality(24), the region $\Delta_{1}$ is assured by the linear control meanwhile the region $\Delta_{2}$ is assured by the NTSM with a fractional power $\kappa$. By selecting proper control parameters such that $k_{1}=k_{2}>\left\|\operatorname{diag}(\boldsymbol{\beta} \sigma) \boldsymbol{J}(\boldsymbol{\eta}) \boldsymbol{M}_{0}^{-1}\right\| \rho(\boldsymbol{e}, \dot{\boldsymbol{e}})$, we have $\Delta<1$. And taking the fact that $1 / \kappa>1$ into consideration, it is obvious that $\Delta_{2}<\Delta_{1}$, which means that the NTSM can achieve higher tracking precision compared with the linear hyperplane-based SMC method.

Remark 5. By replacing the traditional signum function with a fast-TSM-type reaching law, the proposed NTSMC method eliminates the undesirable chattering. However, this operation, in turn, may lead to a subdued robustness against lumped uncertainties compared to the traditional NTSMC method with a signum function term. Meanwhile, it is obvious that the chattering-free nature and the robustness are paradoxical. Thus, to ease the paradox between them, the adaptive methodology is adopted to estimate the lumped uncertainties and reduce the gain of the sign function term.

Therefore, an improved version of the NTSMC method, called adaptive NTSMC (ANTSMC) is proposed in the following subsection.

\subsection{Adaptive Nonsingular Terminal Sliding Mode Control Method Design}

In this subsection, a novel ANTSMC method will be presented. And the corresponding stability will be analyzed using Lyapunov stability theory. 
By combining the same sliding surface and reaching law (21)(22) with the adaptive methodology, the following theorem can be obtained.

Theorem 2. For the 4-DOF simplified dynamic model(5), if assumptions 1 5 holds, and the NTSM and reaching law are chosen as(21)(22), respectively. Then if the controller is designed as follows

$$
\begin{aligned}
\boldsymbol{\tau} & =\boldsymbol{\tau}_{1}+\boldsymbol{\tau}_{2}+\boldsymbol{\tau}_{3}, \\
\boldsymbol{\tau}_{1} & =\boldsymbol{M}_{0} \boldsymbol{J}^{-1}(\boldsymbol{\eta})\left[\ddot{\boldsymbol{\eta}}_{\mathrm{d}}-\dot{\boldsymbol{J}}(\boldsymbol{\eta}) \boldsymbol{v}\right]+\boldsymbol{C}_{0}(\boldsymbol{v}) \boldsymbol{v}+\boldsymbol{D}_{0}(\boldsymbol{v}) \boldsymbol{v}+\boldsymbol{g}_{0}(\boldsymbol{\eta}), \\
\boldsymbol{\tau}_{2} & =-\boldsymbol{M}_{0} \boldsymbol{J}^{-1}(\boldsymbol{\eta}) \operatorname{diag}\left(\boldsymbol{\beta}^{-1} \sigma^{-1}\right)\left[\boldsymbol{k}_{1} \boldsymbol{s}+\boldsymbol{k}_{2} \operatorname{sig}(\boldsymbol{s})^{\kappa}+\operatorname{sig}(\dot{\boldsymbol{e}})^{2-\sigma}\right] . \\
\boldsymbol{\tau}_{3} & =-\boldsymbol{M}_{0} \boldsymbol{J}^{-1}(\boldsymbol{\eta}) \hat{K} \operatorname{sgn}(s) \\
\dot{\hat{K}} & =p[-\varepsilon \hat{K}+|s|]
\end{aligned}
$$

The trajectory of the system will converge to the following ball field in finite time

$$
\left|s_{i}\right| \leq \Omega_{i}=\min \left\{\Omega_{1 i}, \Omega_{2 i}\right\}, \Omega_{1 i}=\left(\frac{\varepsilon_{i} h_{i} \beta_{i} \sigma_{i} K_{i}^{2}}{2\left(1-a_{0 i}\right) k_{2 i}}\right)^{\frac{1}{\kappa_{i}+1}}, \Omega_{2 i}=\left(\frac{\varepsilon_{i} h_{i} \beta_{i} \sigma_{i} K_{i}^{2}}{2\left(1-a_{0 i}\right) k_{1 i}}\right)^{\frac{1}{2}}
$$

Moreover, the position and velocity tracking error of the closed-loop system will converge to the following region in finite time

$$
\left|e_{i}\right| \leq \Delta_{e_{i}}=2 \Omega_{i},\left|\dot{e}_{i}\right| \leq \Delta_{e_{i}}=\left(\beta_{i}^{-1} \Omega_{i}\right)^{1 / \sigma_{i}}
$$

where $\varepsilon=\operatorname{diag}\left(\varepsilon_{1}, \cdots, \varepsilon_{4}\right), \varepsilon_{i}>0$ is positive constant matrix, and $p=\operatorname{diag}\left(p_{1}, \cdots, p_{4}\right), p_{i}>0$ is a positive matrix which can be selected according to (52). And $h_{i}>0.5,0<a_{0 i}<1, i=1 \sim 4$ are positive constants.

Proof. As demonstrated by (Bessa et al., 2010; Slotine et al., 1983), the sliding mode control methodology allows different controller to be separately designed and analyzed for each DOF. Hence, in this proof procedure, we analyze the stability of the closed-loop system in each separate DOF.

Define the estimated error as

$$
\tilde{K}_{i}=K_{i}-\hat{K}_{i}, i=1 \sim 4
$$


where $K_{i}$ is a positive constant, and satisfy $K_{i}>f$.

Choose the Lyapunov function as

$$
V_{i}=s_{i}^{2} / 2+\bar{k}_{i} \tilde{K}_{i}^{2} / 2 p_{i}
$$

where $\bar{k}_{i}=\left[\boldsymbol{\beta} \sigma|\dot{e}|^{\sigma-1}\right]_{i}$. Taking the assumption 1 5 into consideration, we have $\bar{k}_{i} \geq 0$.

Hence, differentiating $V_{i}$ with respect to time along the dynamic model (5) yields

$$
\begin{aligned}
\dot{V}_{i} & =s_{i} \dot{s}_{i}-\bar{k}_{i} \tilde{K}_{i}(-\varepsilon \hat{K}+|s|)_{i}=s_{i}\left[\dot{e}+\bar{k}_{i} \ddot{e}\right]_{i}-\bar{k}_{i} \tilde{K}_{i}|s|_{i}+\varepsilon_{i} \bar{k}_{i} \tilde{K}_{i} \hat{K}_{i} \\
& \left.=s_{i}\left[\dot{e}+\bar{k}_{i}(J \dot{(\eta)})-\ddot{\eta}_{d}+J(\eta) M_{0}^{-1}\left(\tau+F-H_{0}(v, \eta)\right)\right)\right]_{i}-\bar{k}_{i} \tilde{K}_{i}|s|_{i}+\varepsilon_{i} \bar{k}_{i} \tilde{K}_{i} \hat{K}_{i}
\end{aligned}
$$

where $H_{0}(v, \eta)=\boldsymbol{C}_{0}(\boldsymbol{v}) \boldsymbol{v}+\boldsymbol{D}_{0}(\boldsymbol{v}) \boldsymbol{v}+\boldsymbol{g}_{0}(\boldsymbol{\eta})$

Substituting the control law (41) into(46), we have

$$
\dot{V}_{i}=-s_{i}\left[\overrightarrow{\boldsymbol{k}}_{1} \boldsymbol{s}+\overrightarrow{\boldsymbol{k}}_{2} \operatorname{sig}(\boldsymbol{s})^{\kappa}\right]_{i}-\bar{k}_{i} \tilde{K}_{i}|s|_{i}+\varepsilon_{i} \bar{k}_{i} \tilde{K}_{i} \hat{K}_{i}+s_{i} \bar{k}_{i}\left[J(\eta) M_{0}^{-1} F\right]_{i}
$$

where $\overrightarrow{\boldsymbol{k}}_{1}=|\dot{e}|^{\sigma-1} k_{1}, \overrightarrow{\boldsymbol{k}}_{2}=|\dot{e}|^{\sigma-1} k_{2}$.

Taking inequalities (11) and the definition of $\hat{K}_{i}, K_{i}$ into consideration, we have

$$
\dot{V}_{i} \leq-s_{i}\left[\overrightarrow{\boldsymbol{k}}_{1} \boldsymbol{s}+\overrightarrow{\boldsymbol{k}}_{2} \operatorname{sig}(\boldsymbol{s})^{\kappa}\right]_{i}+\varepsilon_{i} \bar{k}_{i} \tilde{K}_{i} \hat{K}_{i}
$$

Therefore, the following two inequalities will hold

$$
\begin{aligned}
& \dot{V}_{i} \leq-\overrightarrow{\boldsymbol{k}}_{2 i}\left|s_{i}\right|^{K_{i}+1}+\varepsilon_{i} \bar{k}_{i} \tilde{K}_{i} \hat{K}_{i} . \\
& \dot{V}_{i} \leq-\overrightarrow{\boldsymbol{k}}_{1 i}\left|s_{i}\right|^{2}+\varepsilon_{i} \bar{k}_{i} \tilde{K}_{i} \hat{K}_{i} .
\end{aligned}
$$

Inequality(49) can be rewritten in the following form as 


$$
\begin{aligned}
\dot{V}_{i} & \leq-\overrightarrow{\boldsymbol{k}}_{2 i}\left|s_{i}\right|^{\kappa_{i}+1}+\varepsilon_{i} \bar{k}_{i} \tilde{K}_{i} \hat{K}_{i} \\
& =-2^{\frac{\kappa_{i}+1}{2}} \overrightarrow{\boldsymbol{k}}_{2 i}\left(\frac{1}{2} s_{i}^{2}\right)^{\frac{\kappa_{i}+1}{2}}-\left(\frac{\varepsilon_{i} \bar{k}_{i}\left(2 h_{i}-1\right)}{2 h_{i}} \tilde{K}_{i}^{2}\right)^{\frac{\kappa_{i}+1}{2}}+\left(\frac{\varepsilon_{i} \bar{k}_{i}\left(2 h_{i}-1\right)}{2 h_{i}} \tilde{K}_{i}^{2}\right)^{\frac{\kappa_{i}+1}{2}}+\varepsilon_{i} \bar{k}_{i} \tilde{K}_{i} \hat{K}_{i} \\
& =-\mu_{i}\left(\left(\frac{1}{2} s_{i}^{2}\right)^{\frac{\kappa_{i}+1}{2}}+\left(\frac{\bar{k}_{i}}{2 p_{i}} \tilde{K}_{i}^{2}\right)^{\frac{\kappa_{i}+1}{2}}\right)+\left(\frac{\varepsilon_{i} \bar{k}_{i}\left(2 h_{i}-1\right)}{2 h_{i}} \tilde{K}_{i}^{2}\right)^{\frac{\kappa_{i}+1}{2}}+\varepsilon_{i} \bar{k}_{i} \tilde{K}_{i} \hat{K}_{i} .
\end{aligned}
$$

where

$$
\mu_{i}=2^{\frac{\kappa_{i}+1}{2}} \overrightarrow{\boldsymbol{k}}_{2 i}, p_{i}=\frac{h_{i} \mu_{i}^{\frac{2}{\kappa_{i}+1}}}{\varepsilon_{i}\left(2 h_{i}-1\right)}
$$

and $h_{i}>\frac{1}{2}$ is a constant.

Here some useful tricks from (Slotine 1983) are applied to inequality(51). According to Lemma 3 and noticing $\frac{1}{2}<\frac{\kappa_{i}+1}{2}<1$, thus if $\frac{\varepsilon_{i} \bar{k}_{i}\left(2 h_{i}-1\right)}{2 h_{i}} \tilde{K}_{i}^{2}>1$, we have

$$
\begin{aligned}
& \left(\frac{\varepsilon_{i} \bar{k}_{i}\left(2 h_{i}-1\right)}{2 h_{i}} \tilde{K}_{i}^{2}\right)^{\frac{\kappa_{i}+1}{2}}+\varepsilon_{i} \bar{k}_{i} \tilde{K}_{i} \hat{K}_{i}<\frac{\varepsilon_{i} \bar{k}_{i}\left(2 h_{i}-1\right)}{2 h_{i}} \tilde{K}_{i}^{2}+\varepsilon_{i} \bar{k}_{i} \tilde{K}_{i} \hat{K}_{i} \leq \frac{\varepsilon_{i} \bar{k}_{i} h_{i}}{2} K_{i}^{2} \\
& \text { If } \frac{\varepsilon_{i} \bar{k}_{i}\left(2 h_{i}-1\right)}{2 h_{i}} \tilde{K}_{i}^{2} \leq 1 \text {, we have } \\
& \left.\left(\frac{\varepsilon_{i} \bar{k}_{i}\left(2 h_{i}-1\right)}{2 h_{i}} \tilde{K}_{i}^{2}\right)^{\frac{\kappa_{i}+1}{2}}\right|_{\frac{\varepsilon_{i} \bar{k}_{i}\left(2 h_{i}-1\right)}{2 h_{i}} \tilde{K}_{i}^{2} \leq 1} ^{<}<\left.\left(\frac{\varepsilon_{i} \bar{k}_{i}\left(2 h_{i}-1\right)}{2 h_{i}} \tilde{K}_{i}^{2}\right)^{\frac{\kappa_{i}+1}{2}}\right|_{\frac{\varepsilon_{i} \bar{k}_{i}\left(2 h_{i}-1\right)}{2 h_{i}} \tilde{K}_{i}^{2}>1}
\end{aligned}
$$

Hence, synthesizing inequalities (53)and(54), we have

$$
\left(\frac{\varepsilon_{i} \bar{k}_{i}\left(2 h_{i}-1\right)}{2 h_{i}} \tilde{K}_{i}^{2}\right)^{\frac{\kappa_{i}+1}{2}}+\varepsilon_{i} \bar{k}_{i} \tilde{K}_{i} \hat{K}_{i} \leq \frac{\varepsilon_{i} \bar{k}_{i} h_{i}}{2} K_{i}^{2}
$$


Then, the inequality (51) can be rewritten as

$$
\dot{V}_{i} \leq-\mu_{i}\left(\left(\frac{1}{2} s_{i}^{2}\right)^{\frac{\kappa_{i}+1}{2}}+\left(\frac{\bar{k}_{i}}{2 p_{i}} \tilde{K}_{i}^{2}\right)^{\frac{\kappa_{i}+1}{2}}\right)+\frac{\varepsilon_{i} \bar{k}_{i} h_{i}}{2} K_{i}^{2} .
$$

According to inequality (13) from Lemma 1 and taking the fact that $\frac{1}{2}<\frac{\kappa_{i}+1}{2}<1$ into consideration, inequality (56) can be rewritten as

$$
\begin{aligned}
\dot{V}_{i} & \leq-\mu_{i}\left(\frac{1}{2} s_{i}^{2}+\frac{\bar{k}_{i}}{2 p_{i}} \tilde{K}_{i}^{2}\right)^{\frac{\kappa_{i}+1}{2}}+\frac{\varepsilon_{i} \bar{k}_{i} h_{i}}{2} K_{i}^{2} \\
& \leq-\mu_{i} V_{i}^{\frac{\kappa_{i}+1}{2}}+\frac{\varepsilon_{i} \bar{k}_{i} h_{i}}{2} K_{i}^{2}
\end{aligned}
$$

Hence, according to Lemma 4, the tracking trajectory of the closed-loop system will converge to the following field in finite time:

$$
\left|s_{i}\right| \leq\left(\frac{\varepsilon_{i} h_{i} \beta_{i} \sigma_{i} K_{i}^{2}}{2\left(1-a_{0 i}\right) k_{2 i}}\right)^{\frac{1}{\kappa_{i}+1}} .
$$

Applying a similar analysis procedure to inequality(50) yields

$$
\left|s_{i}\right| \leq\left(\frac{\varepsilon_{i} h_{i} \beta_{i} \sigma_{i} K_{i}^{2}}{2\left(1-a_{0 i}\right) k_{1 i}}\right)^{\frac{1}{2}}
$$

Thus, synthesizing inequalities (58) and(59), we have that the trajectory of the closed-loop system will be forced into the region (42) in finite time. Similar to the arguments for the proof procedure of Theorem 1, $\dot{e}=0$ will not hinder the reachability of the $\operatorname{NTSM}(21)$. Furthermore, using a similar analysis procedure as was used for the proof of Theorem 1, we can conclude that the position and velocity tracking errors will converge to field(43). This completes the proof. 
Remark 6. Noticing that in(44), the bound information of the lumped uncertainties is needed to select a proper $K_{i}$. However, it is worthy of mention that $K_{i}$ is only used to analyze the stability of the closedloop system and to calculate the tracking error. And as shown in theorem 2, it is not directly used in control law(41).

Remark 7. $p$ is, as shown in equality(52), involved with some other undetermined parameters, which leads to a rather complicated procedure to adopt it. For simplicity, $p$ is often chosen to be large enough to ensure robust stability for practical applications.

Remark 8. Compared with the first control law(23), the second one (41) adopts an adaptive discontinuous term to compensate for the lumped uncertainties. In addition, to ease the chattering problem, a saturation function is often adopted to replace the sign function. As a result, the adaptive term can effectively ease the paradox between chattering and robustness and achieves a better comprehensive control performance.

\section{SIMULATION ANALYSES}

To validate the effectiveness of the proposed control methods, some numerical simulations have been performed on a small ROV named POLARIS showed in Fig.1 using NTSMC and ANTSMC. The nominal physical parameters of the POLARIS ROV are listed in Table 1. $\tau_{f \max }, \tau_{b \max }$, as shown in Table 1, denote the maximum force that one propeller can produce in the forward/backward direction. Additionally, the control parameters of NTSMC and ANTSMC are listed in Table 2. To compare the control performance with the traditional linear hyperplane-based SMC method, we change NTSMC's parameters $\sigma_{i}, \kappa_{i}$ to $\sigma_{i}=1, \kappa_{i}=1$. Then a traditional SMC method can be fairly obtained.

Moreover, the initial position and orientation are $\eta_{0}=-[0.2 m, 0.2 m, 0.2 m, 0.2 \mathrm{rad}]$, and the initial linear velocity and angular velocity are zero. The desired trajectory is selected as $\eta_{d i}=0.1 \sin (0.1 \pi t)$. The 
simulations were performed by using a variable-step forth Dormand-Prince solver with a simple period of $0.1 \mathrm{~s}$.

Two cases were simulated. The first one is to illustrate the trajectory tracking control performance of the proposed methods compared with the traditional SMC method in the presence of lumped uncertainties. The second one is to illustrate the control performance of the ANTSMC method after replacing its signum function term with a saturation function term.

Case one. To validate the robustness of the three controllers against parametric uncertainties and external disturbances, some parametric uncertainties (30\%) are added to the control laws, and some timevarying external disturbances are also added directly into the propeller systems as $\tau_{x, y, z}=0.2 \sin (0.2 \pi \mathrm{t}) \mathrm{N}$ $\tau_{R}=0.05 \sin (0.2 \pi \mathrm{t}) N \cdot \mathrm{m}$. The corresponding simulation results are presented in Fig. 3 to Fig. 6 . And the RMS of the stable tracking error which is from 10 seconds to 40 seconds is listed in Table 3.

It can be observed from the simulation results that all three controllers can provide good robustness against parametric uncertainties and external disturbances, and they can also obtain satisfactory trajectory tracking control performance. Furthermore, it is also obvious that the new proposed ANTSMC method can assure the best control performance among the three controllers. It can provide both the best dynamic response and the best robustness against lumped uncertainties. On the other hand, as shown in Fig.6 the gain vector of $\tau_{3}$ from the ANTSMC method converges to a very small one, which in turn leads to a very smooth control input at the stable stage as shown in Fig.5. However, we can also see a slight chattering in ANTSMC from Fig. 5 in the beginning stage. This is mainly caused by the large tracking error in the initial stage, which leads to a relatively large gain $\hat{K}$ as shown in Fig. 6 and then results in the chattering in the initial stage.

Case two. To ease the chattering in the initial period, the signum function is replaced with a saturation function. A saturation function can be expressed as follows 


$$
\operatorname{sat}\left(\frac{x}{\Delta}\right)=\left\{\begin{array}{l}
\frac{x}{|x|}, \text { for }|x| \geq \Delta . \\
\frac{x}{\Delta}, \text { for }|x| \leq \Delta .
\end{array}\right.
$$

where $\Delta$ is the boundary layer. The saturation function is then used to replace the signum function in the control law (41) with a boundary layer $\Delta_{i}=0.012$. The control performance of the ANTSMC method compared with the other two is presented in Fig.7 to Fig.10 and the RMS of the tracking error in the stable stage is listed in Table 4.

It can be observed clearly from the simulation results that after the replacement operation, the chattering problem of ANTSMC method in the initial stage is effectively eased, as shown in Fig.9. On the other hand, we can see that in this situation, the ANTSMC method can still assure the best trajectory tracking control performance including fastest dynamic response and highest tracking precision.

Broadly speaking, all of the results from case one and case two have sufficiently validated the effectiveness of the two new proposed control methods. Both of them can provide a satisfactory trajectory tracking performance and meanwhile the ANTSMC method can provide even better tracking control performance than the NTSMC method.

\section{CONCLUSIONS}

We have proposed two control methods for the 4-DOF trajectory tracking control problem of ROVs using the NTSMC methodology. Both of them can guarantee satisfactory tracking performance in the presence of parametric uncertainties and external disturbances. Moreover, the adaptive one can provide an even better control performance than the non-adaptive one. The effectiveness of the two methods have been validated through numerical simulations experiments.

\section{ACKNOWLEDGMENTS}

This research was supported by the National Natural Science Foundation of China (Grant No. 
51705243, 51575256), Natural Science Foundation of Jiangsu Province (Grant No. BK20170789) and Open Foundation of the State Key Laboratory of Fluid Power and Mechatronic Systems under Grant No.GZKF-201606.

\section{REFERENCES}

Baek, J., Jin, M., and Han, S 2016. A new adaptive sliding-mode control scheme for application to robot manipulators. IEEE Transactions on Industrial Electronics. 63: 3628-3637.

Bagheri, A., and Moghaddam, J.J 2009. Simulation and tracking control based on neural-network strategy and sliding-mode control for underwater remotely operated vehicle. Neurocomputing. 72: 19341950.

Bessa, W.M., Dutra, M.S., and Kreuzer, E 2008. Depth control of remotely operated underwater vehicles using an adaptive fuzzy sliding mode controller. Robotics and Autonomous Systems. 56: 670677.

Bessa, W.M., Dutra, M.S., and Kreuzer, E 2010. An adaptive fuzzy sliding mode controller for remotely operated underwater vehicles. Robotics and Autonomous Systems. 58: 16-26.

Chu, Z., Zhu, D., and Yang, S. 2017. Observer-based adaptive neural network trajectory tracking control for remotely operated vehicle. IEEE Transactions on Neural Networks and Learning Systems. 28: 16331645.

Cui, R., Chen, L., Yang, C., and Chen, M. 2017. Extended state observer-based integral sliding mode control for an underwater robot with unknown disturbances and uncertain nonlinearities. IEEE Transactions on Industrial Electronics. 64: 6785-6795.

Feng, Y., Yu, X., and Man, Z. 2002. Non-singular terminal sliding mode control of rigid manipulators. Automatica. 38: 2159-2167.

Fossen, T.I. 1994. Guidance and Control of Ocean Vehicles. In New York. Edited by John Wiley and Sons. Oregon State University Press, Corvallis, Oreg. pp. 203-229. 
Hoang, N.Q., and Kreuzer, E 2008. A robust adaptive sliding mode controller for remotely operated vehicles. Technische Mechanik. 3-4: 185-193.

Javadi-Moghaddam, J., and Bagheri, A. 2010. An adaptive neuro-fuzzy sliding mode based genetic algorith control system for under water remotely operated vehicle. Expert Systems With Applications. 00(0): 647-660.

Slotine, J.J.E 1983. Tracking control of nonlinear systems using sliding mode surface. Ph.D. Thesis,Massachusetts Institute of Technology,Cambridge.

Wang, J., and Sun, Z 2012. 6-DOF robust adaptive terminal sliding mode control for spacecraft formation flying. Acta Astronautica. 73: 76-87.

Wang, Y., Gu, L., Gao, M., Jia, X., and Zhu, K. 2014. Studies on nonsingular fast terminal sliding mode control for underwater vehicles. Journal of Zhejiang Univesity (Engineering Science), 10: 1541-1551.

Wang, Y. Gu, L., Chen, B., and Wu, H. 2017. Nonsingular terminal sliding mode control of underwater vehicle-manipulator system based on time delay estimation. Journal of Zhejiang Univesity (Engineering Science), Chinese, in press.

Wang, Y., Gu, L., Gao, M., and Zhu, K. 2016a. Multivariable output feedback adaptive terminal sliding mode control for underwater vehicles. Asian Journal of Control, 18:247-265.

Wang, Y. Gu, L., Xu, Y., and Cao, X. 2016b. Practical tracking control of robot manipulators with continuous fractional-order nonsingular terminal sliding mode. IEEE Transactions on Industrial Electronics. 63: 6194-6204.

Wang, Y. Luo, G., Gu, L, and Li, X. 2016c. Fractional-order nonsingular terminal sliding mode control of hydraulic manipulators using time delay estimation. Journal of Vibration and Control. 22: 3998-4011. Yan, Z., Yu, H., Zhang, W., Li, B., and Zhou, Jia. 2015. Globally finite-time stable tracking control of underactuated UUVs. Ocean Engineering, 107: 132-146.

Yu, S., Yu, X., Shirinzadeh, B., and Man, Z. 2005. Continuous finite-time control for robotic manipulators with terminal sliding mode. Automatica. 41: 1957-1964,. 
Yu, X., Man, Z. 1998. Multi-input uncertain linear systems with terminal sliding-mode control. Automatica. 34: 389-392.

Zhang, M., and Chu, Z. 2012. Adaptive sliding mode control based on local recurrent neural networks for underwater robot. Ocean Engineering. 45: 56-62.

Zhao, D., Zou, T 2012. A finite-time approach to formation control of multiple mobile robots with terminal sliding mode. International Journal of Systems Science. 43: 1998-2014.

Zhu, K., Gu, L. 2011. Multivariable backstepping robust control for work-class remotely operated vehicles. Control Theory \& Applications. 28: 1441-1446.

Zhu, Z., Xia, Y., and Fu, M. 2011. Attitude stabilization of rigid spacecraft with finite-time convergence. International Journal of Robust and Nonlinear Control. 21: 686-702. 


\section{TABLES}

Table 1 Parameters of POLARIS

\begin{tabular}{cccccc}
\hline Parameter & Value & Parameter & Value & Parameter & Value \\
\hline$m / \mathrm{kg}$ & 5.1 & $W / \mathrm{N}$ & 50 & $B / \mathrm{N}$ & 50.4 \\
\hline$Z_{B} / \mathrm{m}$ & -0.05 & $I_{x} /\left(\mathrm{kg} \bullet \mathrm{m}^{2}\right)$ & 0.2 & $I_{y} /\left(\mathrm{kg} \bullet \mathrm{m}^{2}\right)$ & 0.6 \\
\hline$I_{z} /\left(\mathrm{kg} \bullet \mathrm{m}^{2}\right)$ & 0.9 & $X_{\dot{u}} / \mathrm{kg}$ & -3.3 & $Y_{\dot{v}} / \mathrm{kg}$ & -4.3 \\
\hline$Z_{\dot{w}} / \mathrm{kg}$ & -6.4 & $N_{\dot{r}} /\left(\mathrm{kg} \bullet \mathrm{m}^{2}\right)$ & -1.8 & $X_{u} /(\mathrm{kg} / \mathrm{s})$ & -11.2 \\
\hline$Y_{v} /(\mathrm{kg} / \mathrm{s})$ & -13.5 & $Z_{w} /(\mathrm{kg} / \mathrm{s})$ & -16.5 & $N_{r} /(\mathrm{kg} / \mathrm{s})$ & -9.6 \\
\hline$X_{u|u|} /(\mathrm{kg} / \mathrm{m})$ & -3.2 & $Y_{v|v|} /(\mathrm{kg} / \mathrm{m})$ & -2.7 & $Z_{w|w|} /(\mathrm{kg} / \mathrm{m})$ & -3.5 \\
\hline$N_{r|r| r \mid} /(\mathrm{kg} / \mathrm{m})$ & -1.3 & $\tau_{f \max } / \mathrm{N}$ & 1.6 & $\tau_{b \max } / \mathrm{N}$ & -3.2 \\
\hline
\end{tabular}


Table 2. Control parameters of NTSMC and ANTSMC

\begin{tabular}{lccccccc}
\hline & $\beta_{i}$ & $\sigma_{i}$ & $\kappa_{i}$ & $k_{1 i}$ & $k_{2 i}$ & $p_{i}$ & $\varepsilon_{i}$ \\
\hline NTSMC & 2 & 1.4 & $5 / 7$ & 2 & 3 & $*$ & $*$ \\
\hline ANTSMC & 2 & 1.4 & $5 / 7$ & 2 & 3 & 0.1 & 2 \\
\hline
\end{tabular}


Table 3. Root-mean-square of tracking error $\left(\times 10^{-3}\right)(10 \sim 40 \mathrm{~s})$

\begin{tabular}{ccccc}
\hline & $\mathrm{X} / \mathrm{m}$ & $\mathrm{Y} / \mathrm{m}$ & $\mathrm{Z} / \mathrm{m}$ & $\mathrm{R} / \mathrm{rad}$ \\
\hline SMC & 8.2 & 13.2 & 11.5 & 17.0 \\
\hline NTSMC & 4.9 & 9.3 & 5.8 & 10.5 \\
\hline ANTSMC & 4.3 & 8.1 & 5.3 & 9.1 \\
\hline
\end{tabular}


Table 4. Root-mean-square of tracking error $\left(\times 10^{-3}\right)(10 \sim 40 \mathrm{~s})$

\begin{tabular}{ccccc}
\hline & $\mathrm{X} / \mathrm{m}$ & $\mathrm{Y} / \mathrm{m}$ & $\mathrm{Z} / \mathrm{m}$ & $\mathrm{R} / \mathrm{rad}$ \\
\hline SMC & 8.2 & 13.2 & 11.5 & 17.0 \\
\hline NTSMC & 4.9 & 9.3 & 5.8 & 10.5 \\
\hline ANTSMC & 4.5 & 8.2 & 5.4 & 9.3 \\
\hline
\end{tabular}




\section{FIGURES}

Fig. 1. Earth-fixed frame and body-fixed frame

Fig.2 Arrangement of ROVs' propellers

Fig. 3. Trajectory tracking performance under case one

Fig. 4. Tracking error under case one

Fig. 5. Propeller force under case one

Fig. 6. $\hat{K}$ of ANTSMC under case one

Fig. 7. Trajectory tracking performance under case two

Fig. 8. Tracking error under case two

Fig. 9. Propeller force under case two

Fig. 10. $\hat{K}$ of ANTSMC under case two 


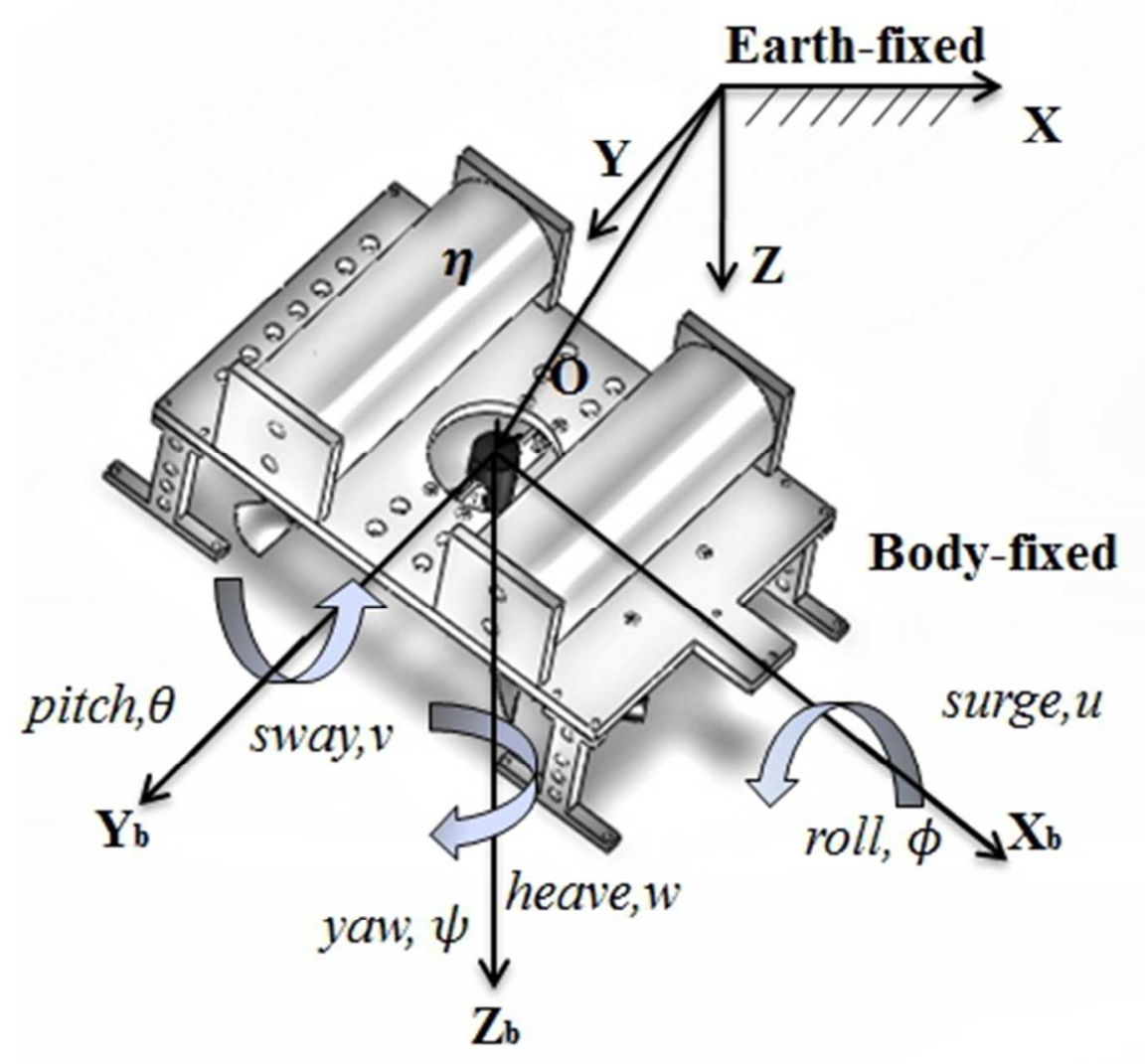

$154 \times 148 \mathrm{~mm}(72 \times 72$ DPI $)$ 


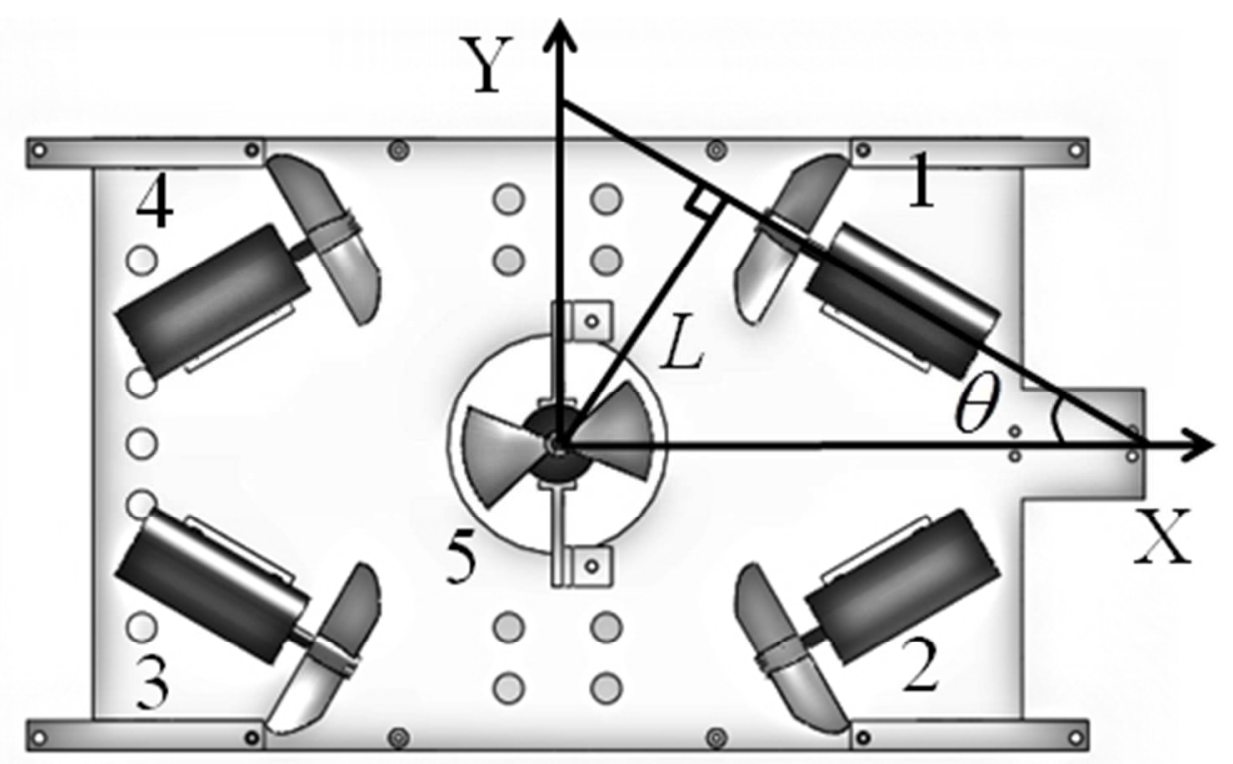

$165 \times 101 \mathrm{~mm}(72 \times 72 \mathrm{DPI})$ 


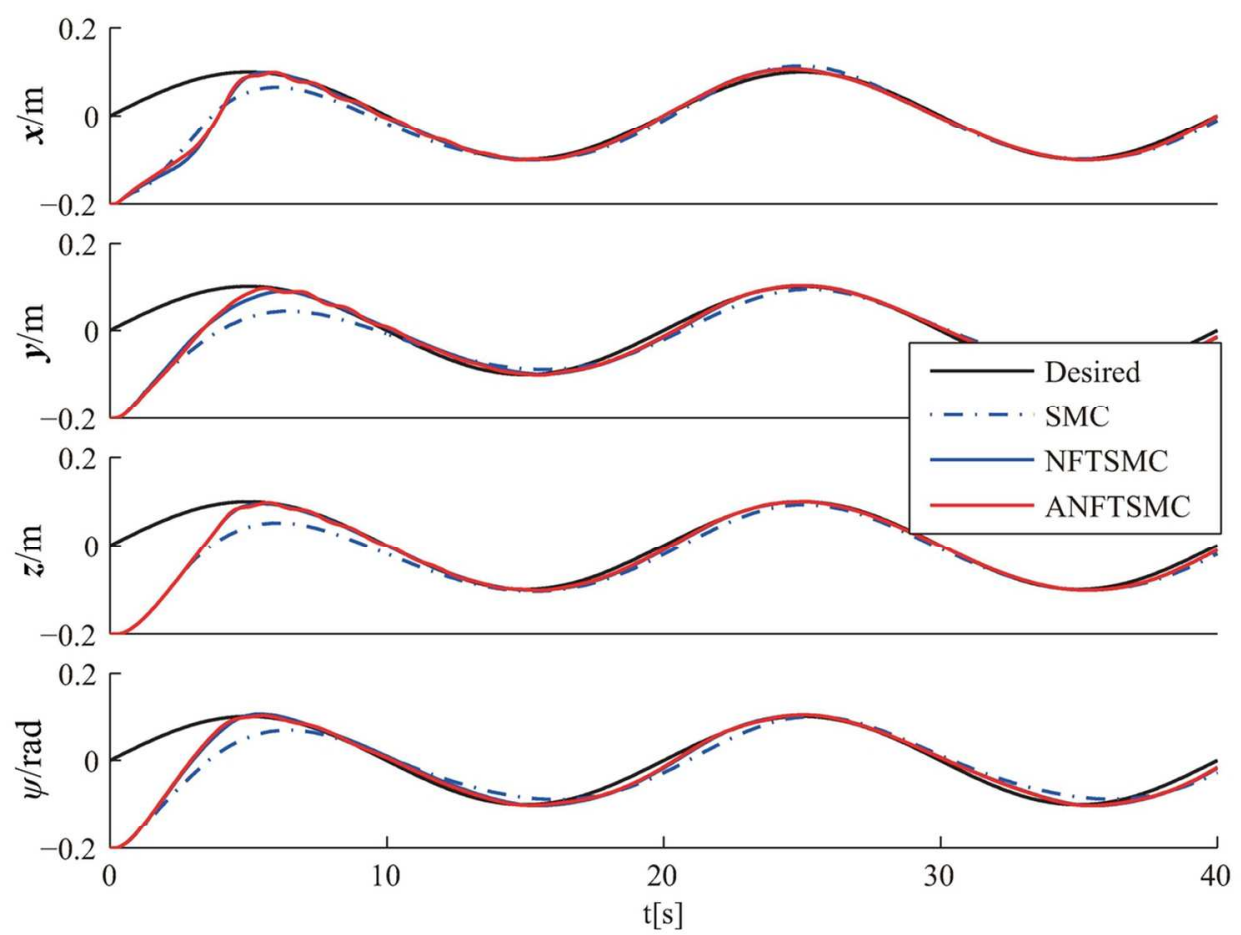

$111 \times 83 \mathrm{~mm}(300 \times 300 \mathrm{DPI})$ 




$132 \times 119 \mathrm{~mm}(300 \times 300 \mathrm{DPI})$ 


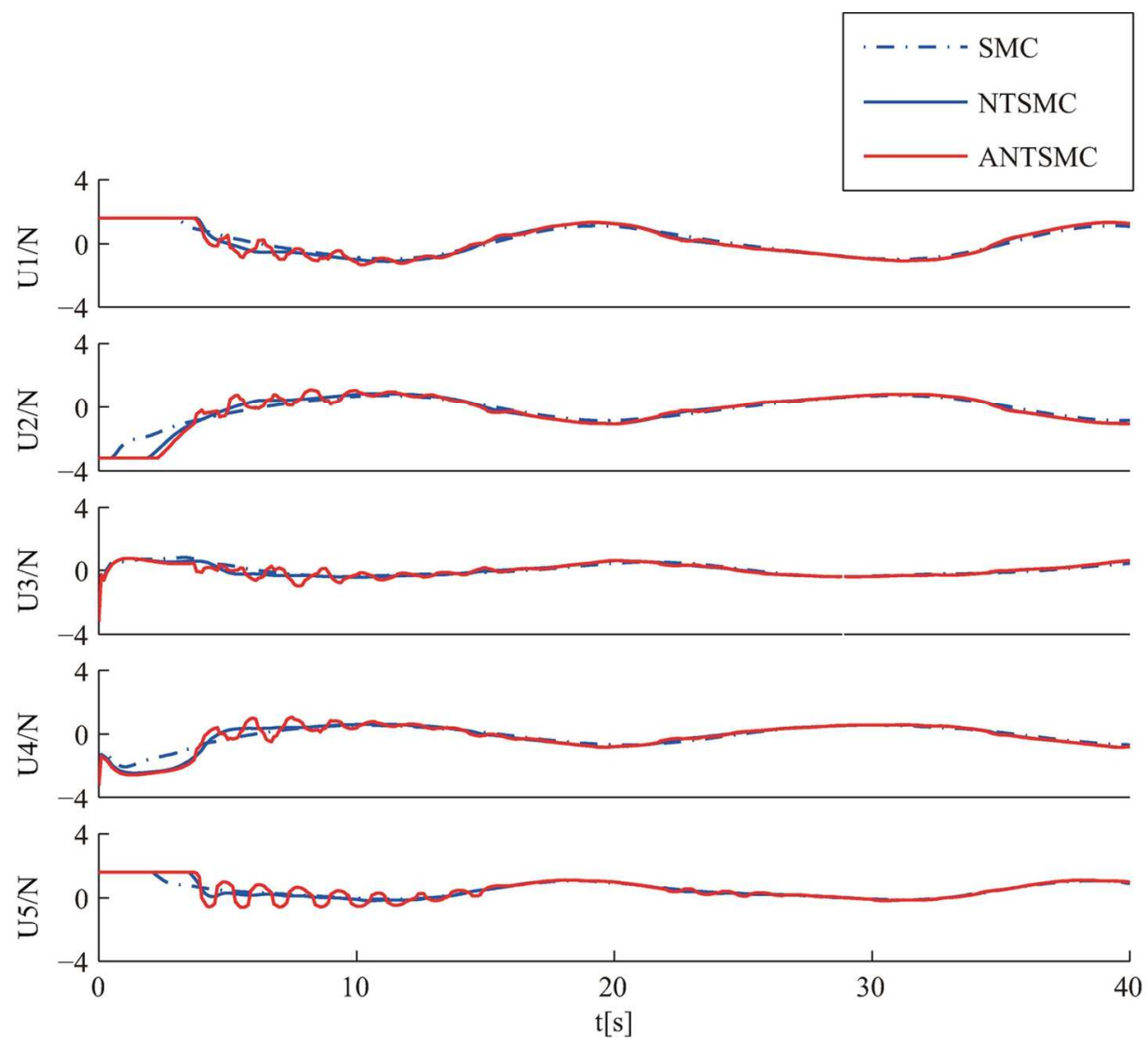

$127 \times 109 \mathrm{~mm}(300 \times 300 \mathrm{DPI})$ 


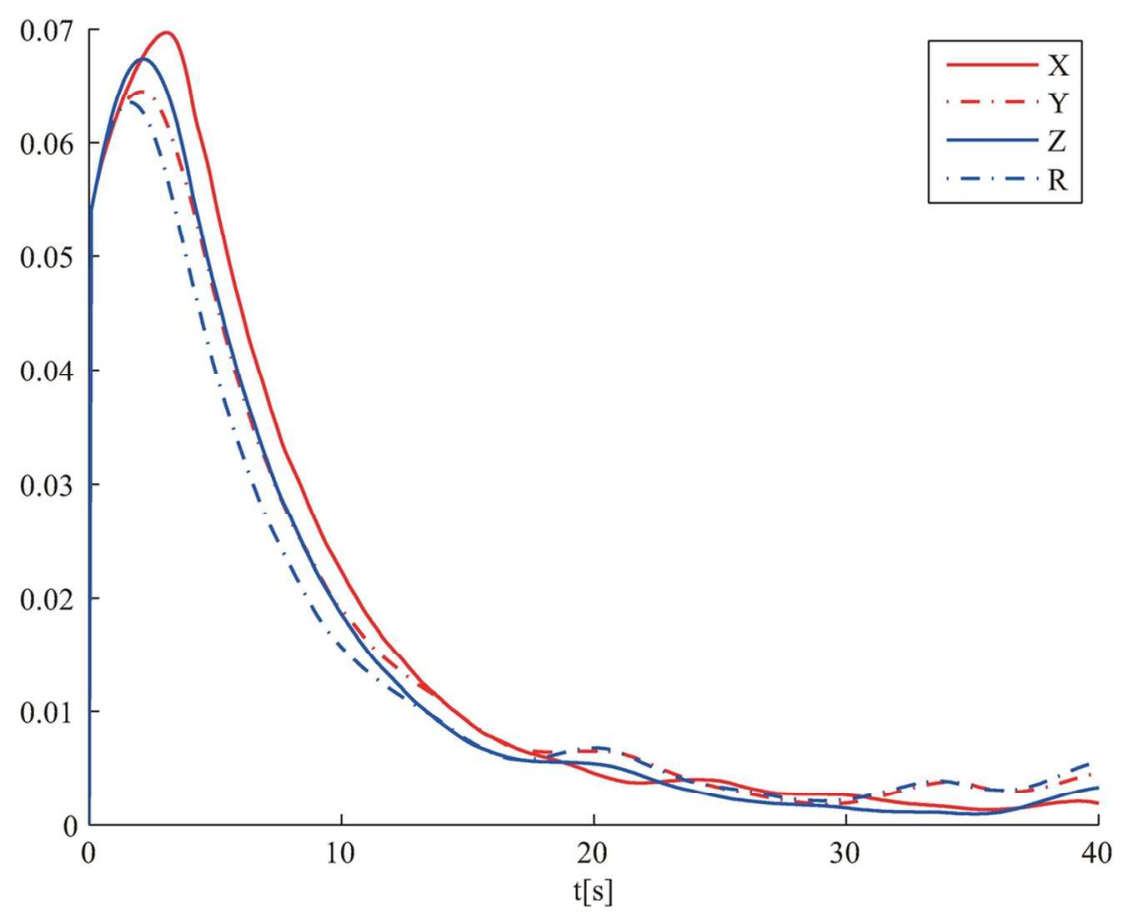

$111 \times 83 \mathrm{~mm}(300 \times 300 \mathrm{DPI})$ 


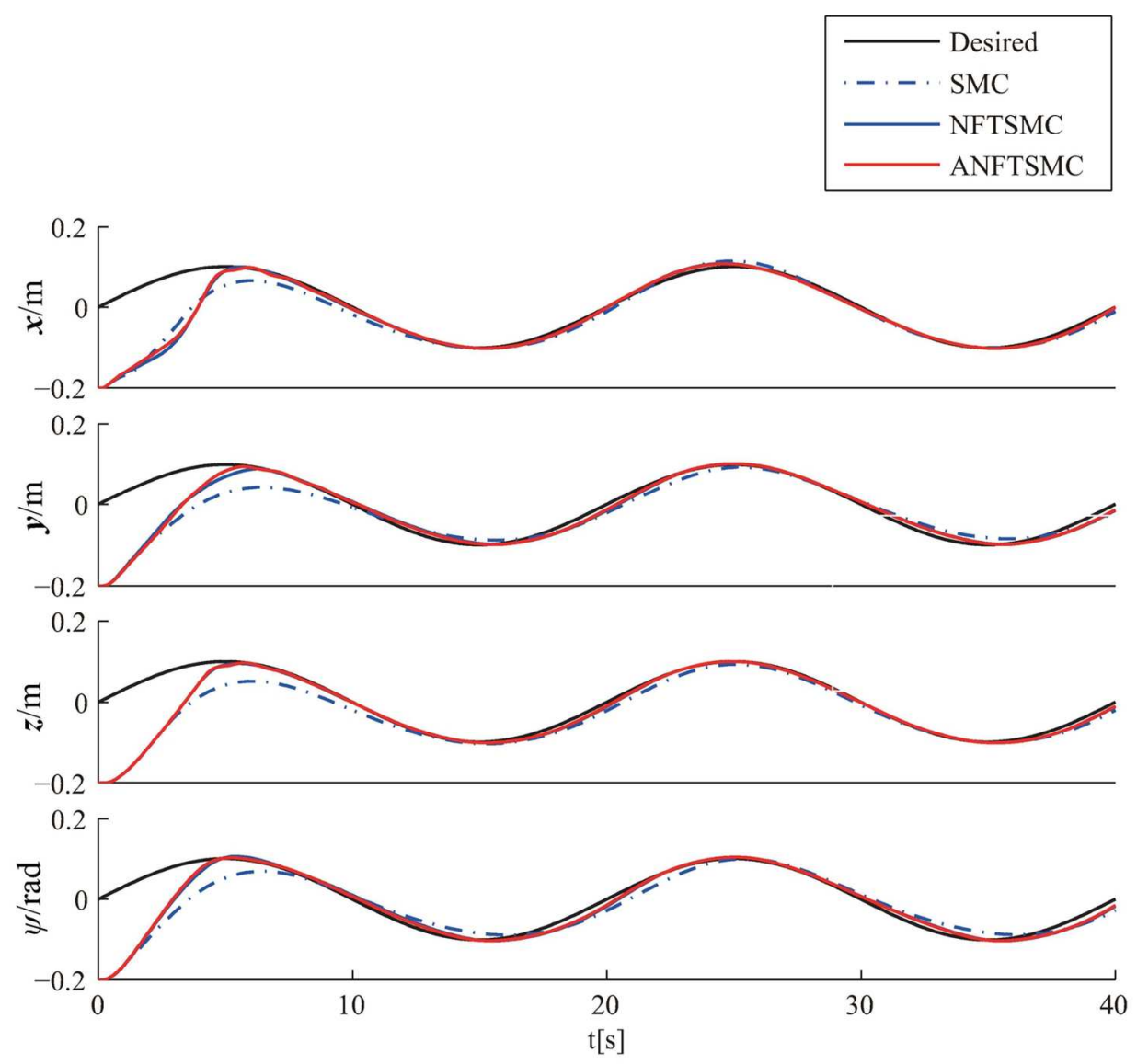

$132 \times 119 \mathrm{~mm}(300 \times 300 \mathrm{DPI})$ 




$135 \times 124 \mathrm{~mm}(300 \times 300$ DPI $)$ 

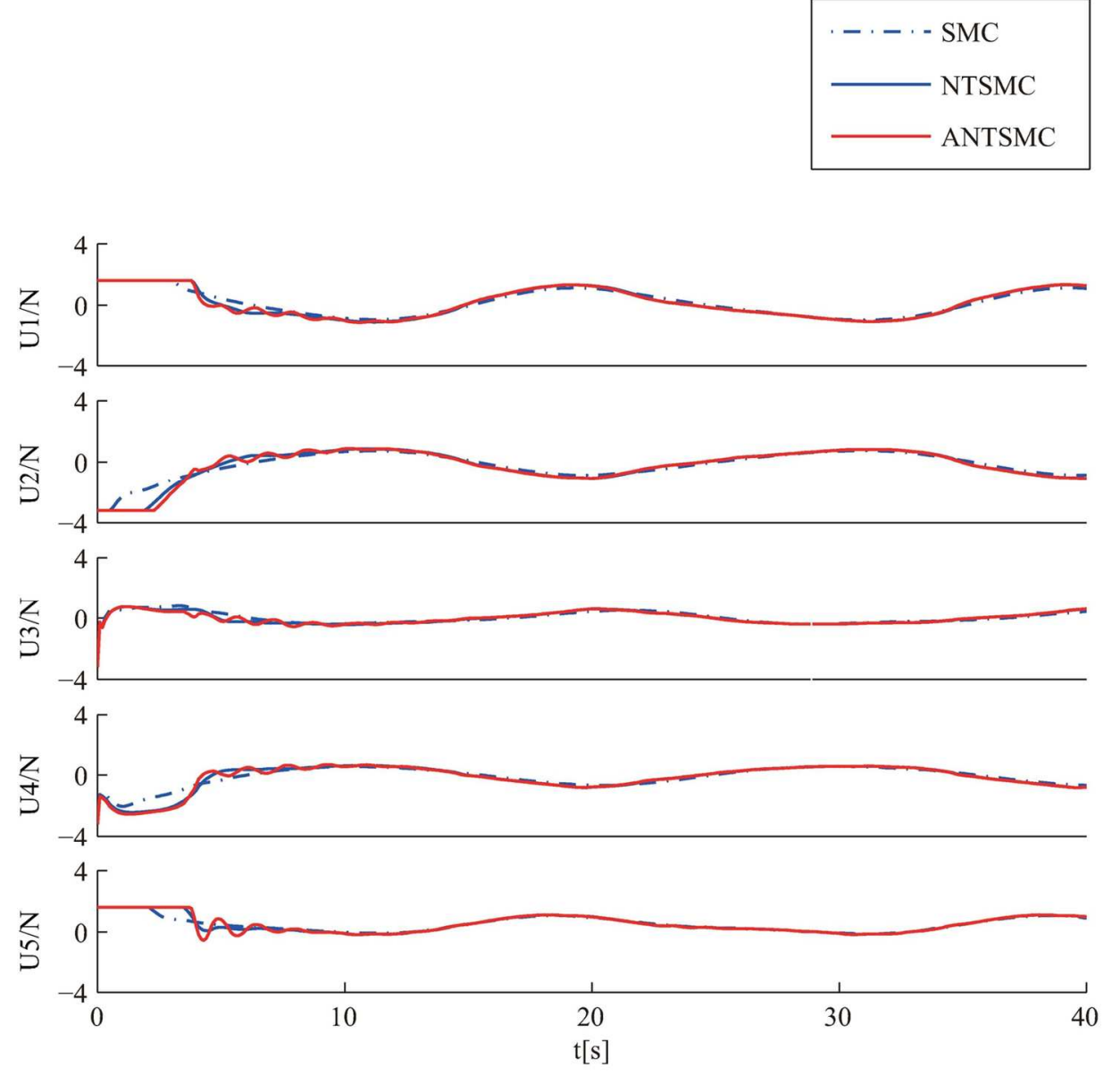

$138 \times 128 \mathrm{~mm}(300 \times 300 \mathrm{DPI})$ 


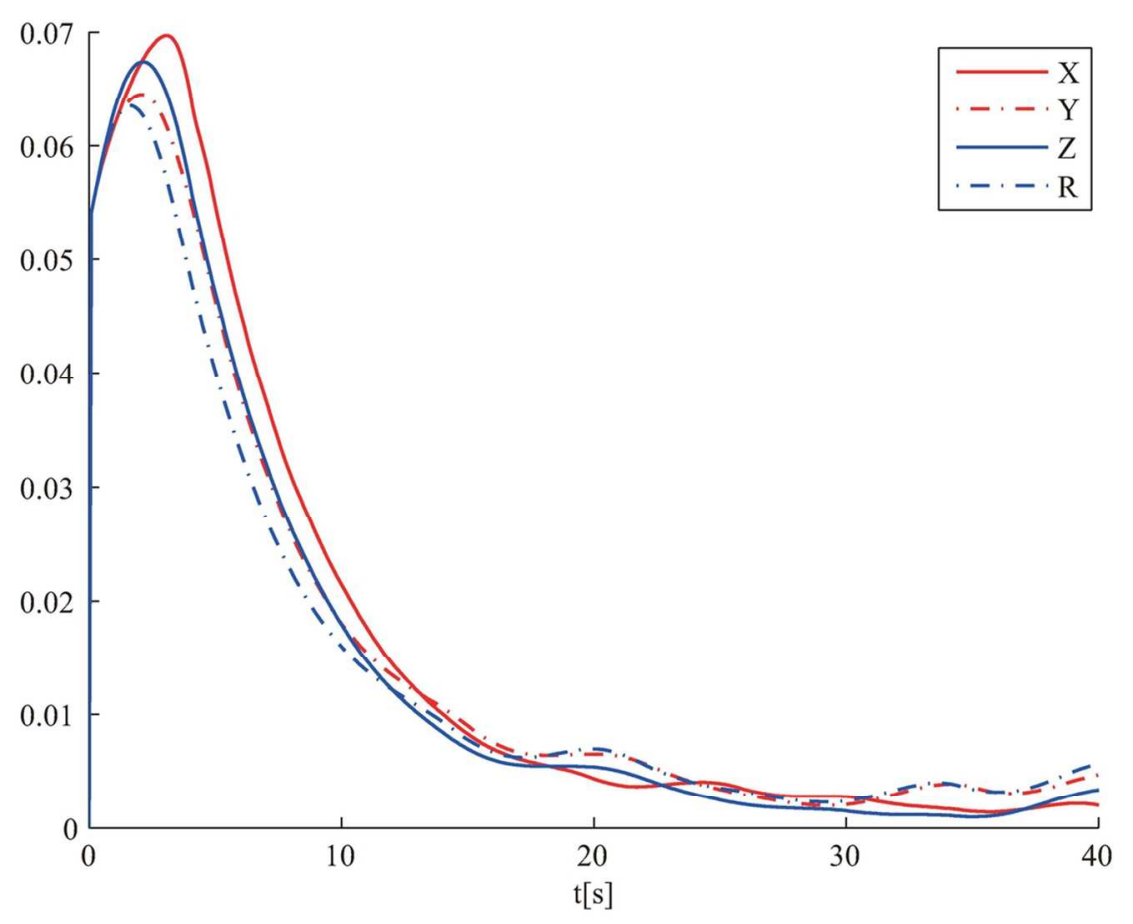

$111 \times 83 \mathrm{~mm}(300 \times 300 \mathrm{DPI})$ 